\title{
Theta Omega Topological Operators and Some Product Theorems
}

\author{
Samer Al Ghour $\mathbb{D}$ and Salma El-Issa $\mathbb{D}$ \\ Department of Mathematics and Statistics, Jordan University of Science and Technology, Irbid 22110, Jordan \\ Correspondence should be addressed to Samer Al Ghour; algore@just.edu.jo
}

Received 6 July 2021; Revised 1 August 2021; Accepted 27 October 2021; Published 16 November 2021

Academic Editor: Ewa Rak

Copyright ( $) 2021$ Samer Al Ghour and Salma El-Issa. This is an open access article distributed under the Creative Commons Attribution License, which permits unrestricted use, distribution, and reproduction in any medium, provided the original work is properly cited.

\begin{abstract}
We introduce and investigate the concepts of $\theta_{\omega}$-limit points and $\theta_{\omega}$-interior points, and we use them to introduce two new topological operators. For a subset $B$ of a topological space $(Y, \sigma)$, denote the set of all limit points of $B$ (resp. $\theta$-limit points of $B$, $\theta_{\omega}$-limit points of $B$, interior points of $B, \theta$-interior points of $B$, and $\theta_{\omega}$-interior points of $\left.B\right)$ by $D(B)\left(\right.$ resp. $D_{\theta}(B), D_{\theta_{\omega}}(B), \operatorname{Int}(B)$, $\operatorname{Int}_{\theta}(B)$, and $\left.\operatorname{Int}_{\theta_{\omega}}(B)\right)$. Several results regarding the two new topological operators are given. In particular, we show that $D_{\theta_{\omega}}(B)$ lies strictly between $D(B)$ and $D_{\theta}(B)$ and $\operatorname{Int}_{\theta_{\omega}}(B)$ lies strictly between $\operatorname{Int}_{\theta}(B)$ and $\operatorname{Int}(B)$. We show that $D(B)=D_{\theta_{\omega}}(B)$ (resp. $\mathrm{Cl}_{\theta}(B)=\mathrm{Cl}_{\theta_{\omega}}(B)$ and $D(B)=D_{\theta_{\omega}}(B)=D_{\theta}(B)$ ) for locally countable topological spaces (resp. antilocally countable topological spaces and regular topological spaces). In addition to these, we introduce several product theorems concerning metacompactness.
\end{abstract}

\section{Introduction}

In 1943, Fomin [1] introduced the notion of $\theta$-continuity. For the purpose of studying the important class of $H$-closed spaces in terms of arbitrary filterbases, the notions of $\theta$-open subsets, $\theta$-closed subsets, and $\theta$-closure were introduced by Velicko [2] in 1966, in which he showed that the family of $\theta$-open sets in a topological space $(Y, \sigma)$ forms a topology on $Y$ denoted by $\sigma_{\theta}$ (see also [3]). The work of Velicko is continued by [3-26] and others. Hdeib [27] introduced the class of $\omega$-closed sets by which he introduced and investigated the notion of $\omega$-continuity. The family of all $\omega$-open sets in $(Y, \sigma)$ is denoted by $\sigma_{\omega}$. It is known that $\sigma_{\omega}$ is a topology on $Y$ which is finer than $\sigma$. Research related to $\omega$-open sets is still a hot area of research [28-36]. In 2017, Al Ghour and Irshidat [37] introduced $\theta_{\omega}$-open subsets, $\theta_{\omega}$-closed subsets, and $\theta_{\omega}$-closure utilizing the topological spaces $\left(Y, \sigma_{\theta}\right)$ and $\left(Y, \sigma_{\omega}\right)$. It is proved in [37] that $\sigma_{\theta_{\omega}}$ forms a topology on $Y$ which lies between $\sigma_{\theta}$ and $\sigma$, and that $\sigma_{\theta_{\omega}}=$ $\sigma$ if and only if $(Y, \sigma)$ is $\omega$-regular. Also, in [37], $\omega-T_{2}$ topological spaces were characterized via $\theta_{\omega}$-open sets. Authors in [35] introduced $\theta_{\omega}$-connectedness and some new separation axioms. Also, research in [37] was continued by various researchers in [28-31]. The notion of interior operators is important in the axiomatization of modal logics.
Judging from the importance of limit points in mathematical analysis, introducing a new limit point notion in any topological structure is still a hot area of research. The first goal of this paper is to introduce and investigate the concepts of $\theta_{\omega}$-limit points and $\theta_{\omega}$-interior points.

In general topology, several topological properties are not finitely productive, such as paracompactness, strong paracompactness, Lindelöfness, and metacompactness. The area of research regarding the problem "What conditions on $(Y, \sigma)$ and $(Z, \delta)$ to insure that their product has property $\mathscr{P}$ "is still hot[38-45]. The second goal of this paper is to introduce several product theorems concerning metacompactness.

\section{Preliminaries}

From now on TS will denote topological space for simplicity. Let $(Y, \sigma)$ and $(Z, \delta)$ be TSs and let $B \subseteq C \subseteq Y$ with $C$ as nonempty. Then, $B$ is called $\omega$-open set in $(Y, \sigma)$ [27] if for each $y \in B$, there is $M \in \sigma$ and a countable set $F \subseteq Y$ such that $y \in M-F \subseteq B$. The relative topology on $C$ is denoted by $\sigma_{C}$, and the product topology on $Y \times Z$ is denoted by $\sigma \times \delta$. The closure of $B$ in $(Y, \sigma)$ (resp. $\left.\left(C, \sigma_{C}\right),\left(Y, \sigma_{\omega}\right)\right)$ is denoted by $\bar{B}$ (resp. $\left.\bar{B}^{C}, \underline{B}_{\omega}\right)$. A point $y \in Y$ is in $\theta$-closure of $B$ [2] $\left(y \in \mathrm{Cl}_{\theta}(B)\right)$ if for every $G \in \sigma$ with $y \in G, \bar{G} \cap B \neq \varnothing$. $B$ is 
called $\theta$-closed [2] if $\mathrm{Cl}_{\theta}(B)=B$. The complement of a $\theta$-closed set is called a $\theta$-open set. It is known that $\sigma_{\theta}=\sigma$ if and only if $(Y, \sigma)$ is regular. A TS $(Y, \sigma)$ is called $\omega$-regular [37] if for each closed set $C$ in $(Y, \sigma)$ and $y \in Y-C$, there exist $G \in \sigma$ and $H \in \sigma_{\omega}$ such that $y \in G, C \subseteq H$, and $G \cap H=\varnothing$. In [37], the author defined $\theta_{\omega}$-closure operator as follows: a point $y \in Y$ is in $\theta_{\omega}$-closure of $B\left(y \in \mathrm{Cl}_{\theta_{\omega}}(B)\right)$ if for any $G \in \sigma$ with $y \in G$ we have $\underline{G}_{\omega} \cap B \neq \varnothing$. $G$ is called $\theta_{\omega}$-closed if $\mathrm{Cl}_{\theta_{\omega}}(G)=G$. The complement of a $\theta_{\omega}$-closed set is called a $\theta_{\omega}$-open set. A TS $(Y, \sigma)$ is called metacompact [46] if every open cover of $(Y, \sigma)$ has a point-finite open refinement.

The following sequence of definitions and theorems will be used in the sequel.

Definition 1 (see [47]). A TS $(Y, \sigma)$ is called locally countable if for each $y \in Y$, there is $G \in \sigma$ such that $G$ is countable and $y \in G$.

Definition 2 (see [48]). A TS $(Y, \sigma)$ is called antilocally countable if each $G \in \sigma-\{\varnothing\}$ is uncountable.

Definition 3 (see [9]). Let $(Y, \sigma)$ be a TS $B \subseteq Y$. A point $y \in Y$ is called $\theta$-limit point of $B$ if for each $G \in \sigma_{\theta}$ with $y \in G$, $G \cap(B-\{y\}) \neq \varnothing$. The set of all $\theta$-limit points of $B$ is called the $\theta$-derived set of $B$ and is denoted by $D_{\theta}(B)$.

Definition 4 (see [9]). Let $(Y, \sigma)$ be a TS and $B \subseteq Y$. A point $y \in Y$ is called a $\theta$-interior point of $B$ if there exists $G \in \sigma$ such that $y \in G \subseteq \bar{G} \subseteq B$. The set of all $\theta$-interior points of $B$ is called the $\theta$-interior of $B$ and is denoted by $\operatorname{Int}_{\theta}(B)$.

Theorem 1 (see [37]). If $(Y, \sigma)$ is locally countable and $B \subseteq Y$, then $\bar{B}=C l_{\theta_{\omega}}(B)$.

Theorem 2 (see [37]). If $(Y, \sigma)$ is antilocally countable and $B \subseteq Y$, then $C l_{\theta}(B)=C l_{\theta_{\omega}}(B)$.

Theorem 3 (see [37]). For any TS $(Y, \sigma), \sigma_{\theta} \subseteq \sigma_{\theta_{\omega}} \subseteq \sigma$.

Theorem 4 (see [2]). A TS $(Y, \sigma)$ is regular if and only if $\sigma=\sigma_{\theta}$.

Theorem 5 (see [37]). Let $(Y, \sigma)$ be a TS and $B \subseteq Y$. Then, $B$ is $\theta_{\omega}$-open set if and only if for each $y \in B$, there exists $G \in \sigma$ such that.

Definition 5. Let $(Y, \sigma)$ and $(Z, \delta)$ be TSs and let $B \subseteq Y$. Then,

(a) $(Y, \sigma)$ is called $C$-scattered if every $B \in \sigma^{c}-\{\varnothing\}$, there is $b \in B$ and a compact set $K$ such that $b \in \operatorname{Int}(K) \subseteq K \subseteq B$ [49]

(b) $B$ is called strongly placed in $Y \times Z$ if for every $z \in Z$ and $H \in \sigma \times \delta$ with $B \times\{z\} \subseteq H$, there are $V \in \sigma$ and $W \in \delta$ such that $B \times\{z\} \subseteq V \times W \subseteq H[50]$

(c) $Y$ is called scattered relative to $Y \times Z$ if for each $B \in \sigma^{c}$, there exists $b \in B$ and $V \in \sigma_{A}$ such that $\bar{V}$ is Lindelöf and strongly placed in $Y \times Z$ [50]
It is well known that if $(Y, \sigma)$ and $(Z, \delta)$ are TSs and $Y$ is $C$-scattered, then $Y$ is scattered relative to $Y \times Z$ but not conversely.

Definition 6 (see [51]). A Hausdorff TS $(Y, \sigma)$ is called ultraparacompact if every open cover of $Y$ has a locally finite clopen refinement.

Ellis [51] showed that a Hausdorff space $(Y, \sigma)$ is ultraparacompact if every open cover has a pairwise disjoint open refinement.

Theorem 6 (see [52]). Let $f:(Y, \sigma) \longrightarrow(Z, \delta)$ be closed and continuous with $(Y, \sigma)$ regular. If $(Z, \delta)$ is metacompact and $f^{-1}(z)$ is Lindelöf for each $z \in Z$, then $(Y, \sigma)$ is metacompact.

Theorem 7 (see [50]). For any two TSs $(Y, \sigma)$ and $(Z, \delta), Y$ is strongly placed in $Y \times Z$ if and only if the projection $\pi:(Y \times$ $Z, \sigma \times \delta) \longrightarrow(Z, \delta)$ is closed.

Theorem 8 (see [35]). Let $(Y, \sigma)$ and $(Z, \delta)$ be TSs and let $B \subseteq Y$. If $B$ is strongly placed in $Y \times Z$ and $C \in \sigma \cap \sigma^{c}$, then $B \cap C$ is strongly placed in $Y \times Z$.

\section{Theta Omega Limit Points}

In this section, we explore the concept of $\theta_{\omega}$-limit points of a set and study its fundamental properties.

Definition 7. Let $(Y, \sigma)$ be a TS and $B \subseteq Y$. A point $y \in Y$ is called $\theta_{\omega}$-limit point of $B$ if for each $G \in \sigma_{\theta_{\omega}}$ with $y \in G$, $G \cap(B-\{y\}) \neq \varnothing$.

The set of all $\theta_{\omega}$-limit points of $B$ is called the $\theta_{\omega}$-derived set of $B$ and is denoted by $D_{\theta_{\omega}}(B)$.

The following result shows that $\theta_{\omega}$-derived set of a set $B$ contains the derived set of $B$ and contained in the $\theta_{\omega}$-derived set of $B$.

Theorem 9. Let $(Y, \sigma)$ be a TS $B \subseteq Y$. The derived set of $B$ is denoted by $D(B)$. Then, $D(B) \subseteq D_{\theta_{\omega}}(B) \subseteq D_{\theta}(B)$.

Proof. To see that $D(B) \subseteq D_{\theta_{\omega}}(B)$, let $y \notin D_{\theta_{\omega}}(B)$, then there exists $G \in \sigma_{\theta}$ such that $y \in G$ and $G \cap(B-\{y\})=\varnothing$. By Theorem $3,{ }^{\omega} G \in \sigma$ and so $y \notin D(B)$. Therefore, we have $D(B) \subseteq D_{\theta_{\omega}}(B)$. To see that $D_{\theta_{\omega}}(B) \subseteq D_{\theta}(B)$, let $y \notin D_{\theta}(B)$, then there exists $G \in \sigma_{\theta}$ such that $y \in G$ and $G \cap(B-\{y\})=\varnothing$. By Theorem 3, $G \in \sigma_{\theta}$ and so $y \notin D_{\theta_{\omega}}(B)$. Therefore, we have $D_{\theta_{\omega}}(B) \subseteq D_{\theta}(B)$.

The following example shows that the equality of each of the inclusions in Theorem 9 does not hold in general.

Example 1 (Example 2.26 of [37]). Let $X=\mathbb{R}$ and let $\sigma=\left\{\varnothing, \mathbb{R}, \mathbb{N}, \mathbb{Q}^{c}, \mathbb{N} \cup \mathbb{Q}^{c}\right\}$. It is proved in [37] that $\sigma_{\theta_{\omega}}=$ $\{\varnothing, \mathbb{R}, \mathbb{N}\}$ and $\sigma_{\theta}=\{\varnothing, \mathbb{R}\}$. Let $B=\{-n: n \in \mathbb{N}\} \cup\{0,1\}$. Then, $D_{\theta}(B)=\mathbb{R}, D_{\theta_{\omega}}(B)=\mathbb{R}-\{1\}$, and $D(B)=\mathbb{Q}-\{1\}$.

Under the condition "regularity," the $\theta_{\omega}$-derived set, the derived set, and the $\theta$-derived set are all equal. 
Theorem 10. Let $(Y, \sigma)$ be a regular $T S$ and $B \subseteq Y$. Then, $D(B)=D_{\theta_{\omega}}(B)=D_{\theta}(B)$.

Proof. It follows from Theorems 3, 4, and 9.

"Local countability" is a sufficient condition for the $\theta_{\omega}$-derived set and the derived set to be equal to each other:

Theorem 11. Let $(Y, \sigma)$ be a locally countable TS and $B \subseteq Y$. Then, $D(B)=D_{\theta_{\omega}}(B)$.

Proof. By Theorem 9, we have $D(B)=D_{\theta}(B)$. To see that $D(B)=D_{\theta_{\omega}}(B)$, suppose to the contrary that there is $y \in D_{\theta_{\omega}}(B)-D(B)$. Since $x \notin D(B)$, there is $G \in \sigma$ such that $G \cap(B-\{y\})=\varnothing$. By Theorem $1, \mathrm{Cl}_{\theta_{\omega}}(Y-G)=\overline{Y-G}=$ $Y-G$ and so $G \in \sigma_{\theta_{\omega}}$. We conclude that $y \notin D_{\theta_{\omega}}(B)$, a contradiction.

"Antilocal countability" is a sufficient condition for the $\theta_{\omega}$-derived set and the $\theta$-derived set to be equal to each other.

Theorem 12. Let $(Y, \sigma)$ be an antilocally countable TS and $B \subseteq Y$. Then, $D_{\theta}(B)=D_{\theta_{\omega}}(B)$.

Proof. By Theorem 9, we have $D_{\theta_{\omega}}(B) \subseteq D_{\theta}(B)$. To see that $D_{\theta}(B) \subseteq D_{\theta_{\omega}}(B)$, suppose to the contrary that there is $y \in D_{\theta}(B)-D_{\theta_{\omega}}(B)$. Since $x \notin D_{\theta_{\omega}}(B)$, there is $G \in \sigma_{\theta_{\omega}}$ such that $G \cap(B-\{y\})=\varnothing$. By Theorem 2, $\mathrm{Cl}_{\theta}(Y-G)=\mathrm{Cl}_{\theta_{\omega}}(Y-G)=Y-G$ and so $G \in \sigma_{\theta}$. We conclude that $y \stackrel{\omega}{\notin} D_{\theta}(B)$, a contradiction.

In Theorems 13-16, we give some natural properties for $\theta_{\omega}$-derived set.

Theorem 13. Let $(Y, \sigma)$ be a TS. If $B \subseteq C \subseteq Y$, then $D_{\theta_{\omega}}(B) \subseteq D_{\theta_{\omega}}(C)$.

Proof. Let $y \notin D_{\theta}(C)$, there exists $G \in \sigma_{\theta}$ such that $y \in G$ and $G \cap(C-\{y\})=\varnothing$. Since $B \subseteq C$, then $G \cap(B-\{x\})=\varnothing$ and hence $y \notin D_{\theta_{\omega}}(B)$. It follows that $D_{\theta_{\omega}}(B) \subseteq D_{\theta_{\omega}}(C)$.

Theorem 14. Let $(Y, \sigma)$ be a TS, and let $A$ and $B$ be subsets of $Y$. Then, $D_{\theta_{\omega}}(A) \cup D_{\theta_{\omega}}(B)=D_{\theta_{\omega}}(A \cup B)$.

Proof. By Theorem 13, $D_{\theta_{\omega}}(B) \subseteq D_{\theta_{u}}(A \cup B)$ and $D_{\theta_{\omega}}(B) \subseteq D_{\theta_{\omega}}(A \cup B)$. Therefore, ${ }^{\prime} D_{\theta_{\omega}}(A) \cup D_{\theta_{\omega}}(B) \subseteq D_{\theta_{\omega}}$ $(A \cup B)$. Now, let $y \notin\left(D_{\theta_{\omega}}(A) \cup D_{\theta}(B)\right)$, then there exist $\theta_{\omega}$-open sets $G, H \in \sigma_{\theta_{\omega}}$ such that $y \in G \cap H$, $(G-\{y\}) \cap A=\varnothing$, and $(H-\{y\}) \cap B=\varnothing$. Let $W=G \cap H$. Then, $W \in \sigma_{\theta_{\omega}}$ and

$$
\begin{aligned}
(W-\{y\}) \cap(A \cup B) & =((W-\{y\}) \cap A) \cup((W-\{y\}) \cap B) \\
& =\varnothing \cup \varnothing \\
& =\varnothing .
\end{aligned}
$$

Thus, $y \notin D_{\theta_{\omega}}(A \cup B)$.
Theorem 15. Let $(Y, \sigma)$ be a TS, and let $A$ and $B$ be subsets of $Y$. Then, $D_{\theta_{\omega}}(A \cap B) \subseteq D_{\theta_{\omega}}(A) \cap D_{\theta_{\omega}}(B)$.

Proof. By Theorem 13, $\quad D_{\theta_{\omega}}(A \cap B) \subseteq D_{\theta_{\omega}}(B) \quad$ and $D_{\theta_{\omega}}(A \cap B) \subseteq D_{\theta_{\omega}}(B)$. Then, $D_{\theta_{\omega}}(A \cap B) \subseteq D_{\theta_{\omega}}(A) \cap D_{\theta_{\omega}}(B)$.

The following example shows that the inclusion in Theorem 15 can not be replaced by equality in general.

Example 2 (Example 2.26 of [37]). Let $Y=\mathbb{R}$ and $\sigma=\left\{\varnothing, \mathbb{R}, \mathbb{N}, \mathbb{Q}^{c}, \mathbb{N} \cup \mathbb{Q}^{c}\right\}$. It is proved in [37] that $\sigma_{\theta}=\{\mathbb{R}, \varnothing, \mathbb{N}\}$. Let $A=((3 / 4),(3 / 2))$ and $B=((5 / 4)$, $(9 / 4))$. Then, $D_{\theta_{\omega}}(A)=\mathbb{R}-\{1\}$ and $D_{\theta_{\omega}}(B)=\mathbb{R}-\{2\}$, so $D_{\theta_{\omega}}(A) \cap D_{\theta_{\omega}}(B)=\mathbb{R}-\{1,2\}$. On the other hand, $D_{\theta_{\omega}}(A \cap B) \stackrel{\stackrel{\omega}{\omega}^{\prime}}{=} D_{\theta_{\omega}}(((5 / 4),(3 / 2)))=\mathbb{R}-\mathbb{N}$.

Theorem 16. Let $(Y, \sigma)$ be a TS and $B \subseteq Y$. Then, $D_{\theta_{\omega}}\left(D_{\theta_{\omega}}(B)\right)-B \subseteq D_{\theta_{\omega}}(B)$.

Proof. Let $y \in D_{\theta_{\omega}}\left(D_{\theta_{\omega}}(B)\right)-B$. Let $G \in \sigma_{\theta_{\omega}}$ with $y \in G$. Since $y \in D_{\theta_{\omega}}\left(D_{\theta_{\omega}}(B)\right), \quad G \cap\left(D_{\theta_{\omega}}(B)-\{y\}\right) \neq \varnothing$. Choose $z \in G \cap\left(D_{\theta_{\omega}}(B)-\{y\}\right)$. Since $z \in D_{\theta_{\omega}}(B)$ and $z \in G \in \sigma_{\theta_{\omega}}$, then $G \cap(B-\{z\}) \neq \varnothing$. Choose $w \in G \cap(B-\{z\})$. Since $w \in B$ and $y \notin B$, then $w \neq y$. Thus, $G \cap(B-\{y\}) \neq \varnothing$ and hence $y \in D_{\theta_{\omega}}(B)$.

The following example shows that the inclusion in Theorem 16 cannot be replaced by equality in general.

Example 3. Let $Y=\mathbb{R}$ and $\sigma=\left\{\mathbb{R}, \varnothing, \mathbb{N}, \mathbb{Q}^{c}, \mathbb{N} \cup \mathbb{Q}^{c}\right\}$. Let $B=((3 / 4),(3 / 2))$. It is proved in [37] that $\sigma_{\theta_{\omega}}=\{\mathbb{R}, \varnothing, \mathbb{N}\}$. By Example 2, $D_{\theta_{\omega}}(B)=\mathbb{R}-\{1\}$. On the other hand,

$$
\begin{aligned}
D_{\theta_{\omega}}\left(D_{\theta_{\omega}}(B)\right)-B & =D_{\theta_{\omega}}(\mathbb{R}-\{1\})-\left(\frac{3}{4}, \frac{3}{2}\right) \\
& =\mathbb{R}-\left(\frac{3}{4}, \frac{3}{2}\right) .
\end{aligned}
$$

\section{Theta Omega Interior Points}

In this section, we explore the concept of $\theta_{\omega}$-interior points of a set and study its fundamental properties.

Definition 8. Let $(Y, \sigma)$ be a TS and $B \subseteq Y$. A point $y \in Y$ is called a $\theta_{\omega}$-interior point of $B$ if there exists $G \in \sigma$ such that $y \in G \subseteq \underline{G}_{\omega} \subseteq B$. The set of all $\theta_{\omega}$-interior points of $B$ is called the $\theta_{\omega}$-interior of $B$ and is denoted by $\operatorname{Int}_{\theta_{\omega}}(B)$.

The following result shows that the $\theta_{\omega}$-interior of a set $B$ contains the $\theta$-interior $B$ and contained in the $\theta_{\omega}$-interior of $B$.

Theorem 17. Let $(Y, \sigma)$ be a TS and $B \subseteq Y$. Then, $\operatorname{Int}_{\theta}(B) \subseteq \operatorname{Int}_{\theta_{\omega}}(B) \subseteq \operatorname{Int}(B)$.

The following example shows that each of the two inclusions in Theorem 17 cannot be replaced by equality in general.

Example 4 (Example 2.26 of [37]). Let $Y=\mathbb{R}$ and let $\sigma=\left\{\varnothing, \mathbb{R}, \mathbb{N}, \mathbb{Q}^{c}, \mathbb{N} \cup \mathbb{Q}^{c}\right\}$. Let $A=\mathbb{N}$ and $B=\mathbb{Q}^{c}$. It is proved in [37] that $\sigma_{\theta_{\omega}}=\{\varnothing, \mathbb{R}, \mathbb{N}\}$ and $\sigma_{\theta}=\{\varnothing, \mathbb{R}\}$. We have 
$\operatorname{Int}_{\theta_{\omega}}(A)=\mathbb{N}$ but $\operatorname{Int}_{\theta}(A)=\varnothing$. Also, we have $\operatorname{Int}_{\theta_{\omega}}(B)=\varnothing$ but $\operatorname{Int}(B)=\mathbb{Q}^{c}$.

Theorem 18. Let $(Y, \sigma)$ be a TS and $B \subseteq Y$. If $G \in \sigma$ such that $G \subseteq \underline{G}_{\omega} \subseteq B$, then $G \subseteq \operatorname{Int}_{\theta_{\omega}}(B)$.

Proof. It follows directly from the definition ofInt $\theta_{\omega}(B)$. $\theta_{\omega}$-interior is always open.

Theorem 19. Let $(Y, \sigma)$ be a TS and $B \subseteq Y$. Then, $\operatorname{Int}_{\theta_{\omega}}(B)$ is $\theta_{\omega}$-open.

Proof. By the definition of $\operatorname{Int}_{\theta}(B)$ and Theorem 18, for every $y \in \operatorname{Int}_{\theta_{\omega}}(B)$, there exists $G_{y} \in \sigma$ such that $y \in G_{y} \subseteq G_{y} \subseteq \operatorname{Int}_{\theta_{\omega}}(B)$. By Theorem 5, it follows that Int $_{\theta}(B) \frac{1}{1 \mathrm{~s}} \theta_{\omega}$-open.

The following is a characterization of $\theta_{\omega}$-open via $\theta_{\omega}$-interior.

Theorem 20. Let $(Y, \sigma)$ be a TS and $B \subseteq Y$. Then, $B$ is $\theta_{\omega}$-open if and only if $B=\operatorname{Int}_{\theta_{\omega}}(B)$.

Proof. Necessity: suppose that $B$ is a $\theta_{\omega}$-open set. By the definition, we have $\operatorname{Int}_{\theta_{\omega}}(B) \subseteq B$. To see that $B \subseteq \operatorname{Int}_{\theta_{\omega}}(B)$, let $y \in B$. By Theorem 5, there exists $G \in \sigma$ such that $y \in G \subseteq \underline{G}_{\omega} \subseteq B$. Then, $y \in \operatorname{Int}_{\theta_{\omega}}(B)$.

Sufficiency: suppose that $B=\operatorname{Int}_{\theta_{\omega}}(B)$. Then, by Theorem $19, B$ is $\theta_{\omega}$-open.

The results in the rest of this section are some natural properties of $\theta_{\omega}$-interior.

Theorem 21. Let $(Y, \sigma)$ be a TS and $B \subseteq Y$. Then, $\operatorname{Int}_{\theta_{\omega}}\left[\operatorname{Int}_{\theta_{\omega}}(B)\right]=\operatorname{Int}_{\theta_{\omega}}(B)$.

Proof. Follows from Theorem 20.

Theorem 22. Let $(Y, \sigma)$ be a TS and $B \subseteq Y$. Then, $Y-\operatorname{Int}_{\theta_{\omega}}(B)=C l_{\theta_{\omega}}(Y-B)$.

Proof. To see that $Y-\operatorname{Int}_{\theta_{\omega}}(B) \subseteq \mathrm{Cl}_{\theta}(Y-B)$, let $y \notin \mathrm{Cl}_{\theta_{\omega}}(Y-B)$. Then, there is $G \in \sigma$ such that $y \in G$ and $\underline{G}_{\omega} \cap(Y-B)=\varnothing$, so we have $y \in G \subseteq \underline{G}_{\omega} \subseteq B$. This shows that $y \notin Y-\operatorname{Int}_{\theta_{\omega}}(B)$. To see that $\mathrm{Cl}_{\theta_{\omega}}(Y-B) \subseteq Y-\operatorname{Int}_{\theta_{\omega}}(B)$, let $y \notin Y-\operatorname{Int}_{\theta_{\omega}}(B)$. Then, $y \in \operatorname{Int}_{\theta_{\omega}}(B)$, and so there is $G \in \sigma$ such that $y \in G \subseteq \underline{G}_{\omega} \subseteq B$. Therefore, we have $\underline{G}_{\omega} \cap(Y-B)=\varnothing$, and so $y \notin \mathrm{Cl}_{\theta_{\omega}}(Y-B)$.

Theorem 23. Let $(Y, \sigma)$ be a TS and $B \subseteq Y$. Then, $Y-C l_{\theta_{\omega}}(B)=\operatorname{Int}_{\theta_{\omega}}(Y-B)$.

Proof. By Theorem 22,

$$
\begin{aligned}
Y-\mathrm{Cl}_{\theta_{\omega}}(B) & =Y-\left(Y-\operatorname{Int}_{\theta_{\omega}}(Y-B)\right) \\
& =\operatorname{Int}_{\theta_{\omega}}(Y-B) .
\end{aligned}
$$

Theorem 24. Let $(Y, \sigma)$ be a TS and let $A \subseteq B \subseteq Y$. Then, $\operatorname{Int}_{\theta_{\omega}}(A) \subseteq \operatorname{Int}_{\theta_{\omega}}(B)$.
Proof. Let $y \in \operatorname{Int}_{\theta_{\omega}}(A)$. Then, there exists $G \in \sigma$ such that $y \in G \subseteq \underline{G}_{\omega} \subseteq A$. Since $A \subseteq B$, then $G \subseteq \bar{U}^{\omega} \subseteq B$. Thus, $y \in \operatorname{Int}_{\theta_{\omega}}(B)$.

Theorem 25. Let $(Y, \sigma)$ be a TS and let $A$ and $B$ be subsets of $Y$. Then, $\operatorname{Int}_{\theta_{\omega}}(A) \cup \operatorname{Int}_{\theta_{\omega}}(B) \subseteq \operatorname{Int}_{\theta_{\omega}}(A \cup B)$.

Proof. By Theorem 24, we have $\operatorname{Int}_{\theta_{\omega}}(A) \subseteq \operatorname{Int}_{\theta_{\omega}}(A \cup B)$ and $\operatorname{Int}_{\theta_{\omega}}(B) \subseteq \operatorname{Int}_{\theta_{\omega}}(A \cup B)$. Thus, $\operatorname{Int}_{\theta_{\omega}}(A) \cup \operatorname{Int}_{\theta_{\omega}}(B) \subseteq \operatorname{Int}_{\theta_{\omega}}$ $(A \cup B)$.

Theorem 26. Let $(Y, \sigma)$ be a TS, and let $A$ and $B$ be subsets of $Y$. Then, $\operatorname{Int}_{\theta_{\omega}}(A \cap B)=\operatorname{Int}_{\theta_{\omega}}(A) \cap \operatorname{Int}_{\theta_{\omega}}(B)$.

Proof. By Theorem 24, we have $\operatorname{Int}_{\theta_{\omega}}(A \cap B) \subseteq \operatorname{Int}_{\theta}(A)$ and Int $_{\theta_{\omega}}(A \cap B) \subseteq \operatorname{Int}_{\theta_{\omega}}(B)$. Thus, $\quad \operatorname{Int}_{\theta}(A \cap B) \subseteq \operatorname{Int}_{\theta^{\omega}}(A) \cap$ $\operatorname{Int}_{\theta_{\omega}}(B)$. To see that $\operatorname{Int}_{\theta_{\omega}}(A) \cap \operatorname{Int}_{\theta_{\omega}}(B) \subseteq \operatorname{Int}_{\theta_{\omega}}(A \cap B)$, let $y \in \operatorname{Int}_{\theta_{\omega}}(A) \cap \operatorname{Int}_{\theta_{\omega}}(B)$. Then, there exist $G, H \in \sigma$ such that $y \in G \subseteq \underline{G}_{\omega} \subseteq A$ and $y \in G \subseteq \underline{H}_{\omega} \subseteq B$. Let $W=G \cap H$. Then, $W \in \sigma$ and $y \in W \subseteq \underline{W}_{\omega}=\underline{G \cap H}_{\omega} \subseteq \underline{G}_{\omega} \cap \underline{H}_{\omega} \subseteq A \cap B$. It follows that $y \in \operatorname{Int}_{\theta_{\omega}}(A \cap B)$.

\section{Metacompactness Product Theorems}

In this section, we introduce several product theorems concerning metacompactness.

The following result will be used in the proof of Theorems 28 and 29.

Theorem 27. Let $(Y, \sigma)$ and $(Z, \delta)$ be metacompact TSs. If for every $y \in Y$ there exists $W \in \sigma$ such that $y \in W$ and $\bar{W} \times$ $Z$ is metacompact, then $(Y \times Z, \sigma \times \delta)$ is metacompact.

Proof. Let $\mathscr{A}$ be an open cover of $(Y \times Z, \sigma \times \delta)$. For every $y \in Y$, choose $W_{y} \in \sigma$ such that $y \in W_{y}$ and $\left(\overline{W_{y}} \times Z,(\sigma \times \delta)_{\overline{W_{y}} \times Z}\right)$ is metacompact. Since $\left\{W_{y}: y \in Y\right\}$ is an open cover of the metacompact TS $(Y, \sigma)$, then it has a point-finite open refinement $\left\{V_{\beta}: \beta \in \Gamma\right\}$. For each $\beta \in \Gamma$, $\overline{V_{\beta}} \times Z,(\sigma \times \delta)_{\overline{V_{\beta}} \times Z}$ is metacompact and has $\mathscr{M}_{\beta}=\left\{A \cap\left(\bar{V}_{\beta} \times Z\right): A \in \mathscr{A}\right\}$ as an open cover, and hence $\mathscr{M}_{\beta}$ has a point-finite open refinement $\mathscr{H}_{\beta}$. It is not difficult to see that $\left\{H \cap\left(V_{\beta} \times Z\right): H \in \mathscr{H}_{\beta}, \beta \in \Gamma\right\}$ is a point-finite open refinement of $\mathscr{A}$. It follows that $(Y \times Z, \sigma \times \delta)$ is metacompact.

The following two product theorems concerning metacompactness will be used in the proof of Theorem 31 which is the main result of this section:

Theorem 28. Let $(Y, \sigma)$ and $(Z, \delta)$ be regular metacompact TSs. If for every $y \in Y$ there exists $W \in \sigma$ such that $y \in W, \bar{W}$ is strongly placed in $Y \times Z$, and $\left(\bar{W}, \sigma_{\bar{W}}\right)$ is Lindelöf, then $(Y \times Z, \sigma \times \delta)$ is metacompact.

Proof. For each $y \in Y$, choose $W_{y} \in \sigma$ such that $y \in W, \bar{W}$ is strongly placed in $Y \times Z$, and $\left(W_{y}, \sigma_{\overline{W_{\gamma}}}\right)$ is Lindelöf. For every $y \in Y, \bar{W}_{y}$ is strongly placed in $Y \times Z$ and so by Theorem 6 , the projection function $\pi_{y}:\left(\overline{W_{y}} \times Z, \quad(\sigma\right.$ $\left.\times \delta)_{\overline{W_{y}} \times Z}\right) \longrightarrow(Z, \delta)$ is a closed function. For every $y \in Y$, $\left(\overline{W_{y}}, \sigma_{\overline{W_{y}}}\right)$ is Lindelöf and since $\pi_{y}^{-1}(z)=\overline{W_{y}} \times\{z\}$, then 
$\left(\overline{W_{y}} \times\{z\},(\sigma \times \delta)_{\overline{W_{y}} \times\{z\}}\right)$ is Lindelöf. For every $y \in Y$, $\left(\overline{W_{y}}, \sigma_{\overline{W_{y}}}\right)$ is metacompact and so by Theorem 6 , $\left(\overline{W_{y}} \times\right.$ $\left.Z,(\sigma \times \delta)_{\overline{W_{y}} \times Z}\right)$ is metacompact. Thus, by Theorem 27, we have $(Y \times Z, \sigma \times \delta)$ is metacompact.

Theorem 29. Let $(Y, \sigma)$ and $(Z, \delta)$ be metacompact TSs and let $B \subseteq Y$ such that $B$ is closed in $(Y, \sigma),\left(B, \sigma_{B}\right)$ is Lindelöf, and $B$ is strongly placed in $Y \times Z$. If for all $y \in Y-B$ there is $M \in \sigma_{Y-B}$ such that $\left(\bar{M}^{Y-B} \times Z,(\sigma \times \delta)_{\bar{M}^{Y-B} \times Z}\right)$ is metacompact, then $(Y \times Z, \sigma \times \delta)$ is metacompact.

Proof. Let $\mathscr{A}$ be an open cover of $(Y \times Z, \sigma \times \delta)$. For each $z \in Z, \quad\left(M \times\{z\},(\sigma \times \delta)_{M \times\{z\}}\right) \quad$ is Lindelöf with $M \times\{z\} \subset \bigcup \mathscr{A}$, and so there exists $\mathscr{A}_{z} \subseteq \mathscr{A}$ such that $\mathscr{A}$ is countable and $M \times\{z\} \subseteq \bigcup \mathscr{A}_{z}$. Since $M$ is strongly places in $Y \times Z$, then for every $z \in Z$, there exist $U_{z} \in \sigma$ and $V_{z} \in \delta$ such that $M \times\{z\} \subseteq U_{z} \times V_{z} \subseteq \bigcup \mathscr{A}_{z}$. Since $\left\{V_{z}: z \in Z\right\}$ is an open cover of the metacompact TS $(Z, \delta)$, then it has a point-finite open refinement $\left\{G_{\beta}: \beta \in \Gamma\right\}$. For each $\beta \in \Gamma$, choose $z(\beta)$ such that $G_{\beta} \subseteq V_{z(\beta)}$. Then, by Theorem 27 and the assumption, it is not difficult to see that $\left(\left(Y-U_{z(\beta)}\right) \times\right.$ $\left.Z,(\sigma \times \delta)_{\left(Y-U_{z(\beta)}\right) \times Z}\right)$ is metacompact. Since $\left\{A \cap\left(\left(Y-U_{z(\beta)}\right) \times Z\right): A \in \mathscr{A}\right\}$ is an open cover of $\left(Y-U_{z(\beta)}\right) \times Z$, then it has a point-finite open refinement $\mathscr{A}_{\beta}$. It is not difficult to check that

$$
\begin{gathered}
\left\{A \cap\left(U_{z(\beta)} \times G_{\beta}\right): \quad A \in \mathscr{A}_{z(\beta)}, \beta \in \Gamma\right\} \\
\cup\left\{G \cap\left(Y \times G_{\beta}\right): \quad G \in \mathscr{A}_{\beta}, \beta \in \Gamma\right\},
\end{gathered}
$$

is a point-finite open refinement of $\mathscr{A}$. Therefore, $(Y \times Z, \sigma \times \delta)$ is metacompact.

Theorem 30. Let $(Y, \sigma)$ be ultraparacompact and $(Z, \delta)$ be metacompact. Suppose there exists $D \subseteq Y$ such that $D$ is closed in $(Y, \sigma)$ and for every $y \in D$ there exists $W \in \sigma_{D}$ such that $y \in W, \bar{W}$ is strongly placed in $Y \times Z$, and $\left(\bar{W}, \sigma_{\bar{W}}\right)$ is Lindelöf, and for every $y \in Y-D$, there is $K \in \sigma_{Y-D}$ such that $y \in K$ and $\bar{K}^{Y-D} \times Y$ is metacompact. Then, $(Y \times Z, \sigma \times \delta)$ is metacompact.

Proof. By assumption there exists $\mathscr{A} \subseteq \sigma_{D}$ such that for all $A \in \mathscr{A}, \bar{A}$ is strongly placed in $Y \times Z$ and $\left(\bar{A}, \sigma_{\bar{A}}\right)$ is Lindelöf, and $\bigcup \mathscr{A}=D$. Since $\left(D, \sigma_{D}\right)$ is ultraparacompact, then $\mathscr{A}$ has a pairwise disjoint open refinement $\left\{C_{\beta}: \beta \in \Gamma\right\} \subseteq \sigma_{D}$. For every $\beta \in \Gamma$, choose $U_{\beta} \in \sigma$ such that $C_{\beta}=U_{\beta} \cap D$. Put $\mathscr{H}=\left\{U_{\beta}: \beta \in \Gamma\right\} \cup\{Y-D\}$. Since $(Y, \sigma)$ is ultraparacompact and $\mathscr{H}$ is an open cover of $(Y, \sigma)$, then $\mathscr{H}$ has a pairwise disjoint open refinement $\left\{M_{\gamma}: \gamma \in \Delta\right\}$. For every $\gamma \in \Delta, M_{\gamma}$ meets at most one member of $\left\{C_{\beta}: \beta \in \Gamma\right\}$. For every $\quad \gamma \in \Delta, \quad$ let $\left(M_{\gamma}\right)^{*}=M_{\gamma} \cap\left(\cup\left\{C_{\beta}: \beta \in \Gamma\right.\right.$ and $\left.\left.C_{\beta} \cap M_{\gamma} \neq \varnothing\right\}\right)$, then $\left(M_{\gamma}\right)^{*}=\varnothing$ or $\left(M_{\gamma}\right)^{*}=M_{\gamma} \cap \bar{A}$; for some $A \in \mathscr{A}$, it follows that $\left(M_{\gamma}\right)^{*}$ is closed in $\left(M_{\gamma}, \sigma_{M_{\gamma}}\right)$ and $\left(\left(M_{\gamma}\right)^{*}, \sigma_{\left(M_{\gamma}\right)^{*}}\right)$ is Lindelöf and by Theorem 8 ; it is strongly placed in $M_{\gamma} \times Y$. By the assumption on $Y-$ $D$ and Theorem 29, we conclude that $\left(M_{\gamma} \times Z,(\sigma \times \delta)_{M_{\gamma} \times Y}\right)$ is metacompact. Since $Y \times Z=\cup_{\gamma \in \Delta} M_{\gamma} \times Z$ is metacompact, then $(Y \times Z, \sigma \times \delta)$ is metacompact.
Now, we are ready to state the main result of this section.

Theorem 31. Let $(Y, \sigma)$ be ultraparacompact and $(Z, \delta)$ be regular and metacompact such that $Y$ is scattered relative to the product $Y \times Z$, then $(Y \times Z, \sigma \times \delta)$ is metacompact.

Proof. Denote by $Y^{(0)}=Y$ and $Y^{(1)}=\{y \in Y$ : there is no $U \in \sigma$ such that $y \in U$ and $\bar{U}$ is strongly placed in $Y \times Z$ and closure $\left(\bar{U}, \sigma_{\bar{U}}\right)$ is Lindelöf $\}$. If there is an ordinal $\alpha>1$ such that $Y^{(\alpha)}$ has been defined and $\beta=\alpha+1$, then $Y^{(\beta)}=\left(Y^{(\alpha)}\right)^{(1)}$. If $\alpha$ is a limit ordinal, then $Y^{(\alpha)}=\cap_{\beta<\alpha} Y^{(\beta)}$. Since $Y$ is scattered relative to $Y \times Z$, then there exists an ordinal $\alpha$ such that $Y^{(\alpha)}=\varnothing$.

The proof proceeds by transfinite induction on $\alpha$. If $Y^{(1)}=\varnothing$, then for every $y \in Y$ there exists $U_{y} \in \sigma$ such that $y \in U$ and $\bar{U}$ is strongly placed in $Y \times Z$ and closure $\left(\bar{U}, \sigma_{\bar{U}}\right)$. And by Theorem 28, $(Y \times Z, \sigma \times \delta)$ is metacompact. If $Y^{(\alpha+1)}=\varnothing$, then for every point $y \in Y^{(\alpha)}$ there exists $U_{y} \in \sigma_{Y^{(\alpha)}}$ such that $y \in U_{y}, \overline{U_{y}}$ is strongly placed in $Y \times Z$, and $\left(\bar{U}_{y}, \sigma_{U_{y}}\right)$ is Lindelöf and if $y \in Y-Y^{(\alpha)}$, choose a clopen set $C_{y}$ such that $y \in C_{y} \subseteq Y-Y^{(\alpha)}$. Since $C_{y}^{(\alpha)} \subseteq\left(Y-Y^{(\alpha)}\right)^{(\alpha)}=\varnothing$, then $C_{y}$ is scattered relative to $C_{y} \times$ $Z$ and $\left(C_{y}, \sigma_{C_{y}}\right)$ is ultraparacompact, and by the inductive assumption, it follows that $\left(C_{y} \times Z,(\sigma \times \delta)_{C_{y} \times Z}\right)$ is metacompact.

If $Y^{(\alpha)}=\varnothing$ for the limit ordinal $\alpha$, then the open cover $\left\{Y-Y^{(\beta)}: \beta<\alpha\right\}$ has a pairwise disjoint open refinement $\left\{O_{\gamma}: \gamma \in \Gamma\right\}$. For each $\gamma \in \Gamma$, choose $\beta<\alpha$ such that $O_{\gamma} \subseteq Y-Y^{(\beta)}$. Therefore, $\left(O_{\gamma}\right)^{(\beta)}=\varnothing$, and hence $\left(O_{\gamma} \times Z\right.$, $\left.(\sigma \times \delta)_{O_{\gamma} \times Z}\right)$ is metacompact. Since $Y \times Z=\cup_{\gamma \in \Gamma} O_{\gamma} \times Y$, it follows that $(Y \times Z, \sigma \times \delta)$ is metacompact.

Corollary 1. The product of an ultraparacompact C-scattered TS with a metacompact regular $T S$ is again metacompact.

By the end of this paper, the authors found it is suitable to raise the following open question.

Question 1. Let $(Y, \sigma)$ and $(Z, \delta)$ be regular and metacompact TSs such $Y$ is scattered relative to the product $Y \times Z$. Is $(Y \times Z, \sigma \times \delta)$ metacompact?

\section{Conclusion}

In this work, the research via $\theta_{\omega}$-open sets is continued by introducing the notions of $\theta_{\omega}$-limit points and $\theta_{\omega}$-interior points. Several relationships regarding these two notions are introduced. Moreover, several product theorems concerning metacompactness are given. In future studies, the following topics could be considered: (1) define $\theta_{\omega}$-border, $\theta_{\omega}$-frontier, and $\theta_{\omega}$-exterior of a set using $\theta_{\omega}$-open sets and (2) try to solve Question 1.

\section{Data Availability}

No data were used to support this study. 


\section{Conflicts of Interest}

The authors declare that they have no conflicts of interest.

\section{References}

[1] S. Fomin, "Extensions of topological spaces," Annals of Mathematics, vol. 44, no. 3, pp. 471-480, 1943.

[2] N. V. Velicko, "H-closed topological spaces," Matematicheskii Sbornik, vol. 70, pp. 98-112, 1966.

[3] P. E. Long and L. L. Herrington, "The $\tau_{\theta}$-topology and faintly continuous functions," Kyungpook Mathematical Journal, vol. 22, pp. 7-14, 1982.

[4] R. Dickman Jr. and J. Porter, " $\theta$-closed subsets of Hausdorff spaces," Pacific Journal of Mathematics, vol. 59, no. 2, pp. 407-415, 1975.

[5] R. F. Dickman Jr. and J. R. Porter, " $\theta$-perfect and $\theta$-absolutely closed functions," Illinois Journal of Mathematics, vol. 21, pp. 42-60, 1977.

[6] J. E. Joseph, " $\theta$-closure and $\theta$-subclosed graphs," Mathematical Chronicle, vol. 8, pp. 99-117, 1979.

[7] T. Noiri and S. Jafari, "Properties of $(\theta, s)$-continuous functions," Topology and its Applications, vol. 123, no. 1, pp. 167-179, 2002.

[8] M. Mrsevic and D. Andrijevic, "On $\theta$-connectedness and $\theta$-closure spaces," Topology and its Applications, vol. 123, no. 1, pp. 157-166, 2002.

[9] M. Caldas, S. Jafari, and M. M. Kovar, "Some properties of $\theta$-open sets," Divulgaciones Matematicas, vol. 12, no. 2, pp. 161-169, 2004.

[10] Á. Császár, "Further separation properties of $\theta$-modifications," Acta Mathematica Hungarica, vol. 102, no. 3, pp. 249-260, 2004.

[11] M. Caldas, D. N. Georgiou, S. Jafari, and T. Noiri, "On $(\Lambda, \theta)$-closed sets," Questions and Answers in General Topology, vol. 23, pp. 69-87, 2005.

[12] J. Cao, M. Ganster, I. L. Reilly, and M. Steiner, " $\delta$-closure, $\theta$-closure and generalized closed sets," Applied General Topology, vol. 6, no. 1, pp. 79-86, 2005.

[13] C. K. Basu, M. K. Ghosh, and S. S. Mandal, "A generalization of H-closed spaces," Tamkang Journal of Mathematics, vol. 39, no. 2, pp. 143-154, 2008.

[14] Á. Császár and E. Makai Jr., "Further remarks on $\delta$ - and $\theta$-modifications," Acta Mathematica Hungarica, vol. 123, no. 3, pp. 223-228, 2009.

[15] E. Ekici, S. Jafari, and R. M. Latif, "On a finer topological space than $\tau_{\theta}$ and some maps," Italian Journal of Pure and Applied Mathematics, vol. 27, pp. 293-304, 2010.

[16] A. Caserta and G. Di Maio, "Variations on selective separability in non-regular spaces," Topology and its Applications, vol. 160 , no. 18 , pp. 2379-2385, 2013.

[17] M. Alena Chernikava and A. Chernikava, "On the proof of the existence of undominated strategies in normal form games," The American Mathematical Monthly, vol. 121, no. 4, pp. 332-337, 2014.

[18] A. Pavlovic, "Local function versus local closure function in ideal topological spaces," Filomat, vol. 30 , no. 14, pp. 3725-3731, 2016.

[19] S. Ozcag, "Selective bitopological versions of separability," Topology and its Applications, vol. 201, pp. 403-413, 2016.

[20] I. S. Gotchev, "The non-Urysohn number of a topological space," Houston Journal of Mathematics, vol. 43, no. 1, pp. 223-235, 2017.
[21] J. D. Reyes and A. R. Morales, "The weak Urysohn number and upper bounds for cardinality of Hausdorff spaces," Houston Journal of Mathematics, vol. 44, no. 4, pp. 1389-1398, 2018.

[22] A. Ramírez-Páramo, "A generalization of some cardinal function inequalities," Topology and its Applications, vol. 256, pp. 228-234, 2019.

[23] L. Babinkostova, B. A. Pansera, and M. Scheepers, "Selective versions of $\theta$-density," Topology and its Applications, vol. 258, pp. 268-281, 2019.

[24] P. Periyasamy and P. Rock Ramesh, "Local $\delta$-closure functions in ideal topological spaces," Advances in Mathematics: Scientific Journal, vol. 9, no. 5, pp. 2427-2436, 2020.

[25] A. V. Osipov, "On the cardinality of S(n)-Spaces," Quaestiones Mathematicae, vol. 44, no. 1, pp. 121-128, 2021.

[26] J. A. Hassan and M. A. Labendia, " $\theta_{s}$-open sets and $\theta_{s}$-continuity of maps in the product space," The Journal of Mathematics and Computer Science, vol. 25, no. 2, pp. 182190, 2022.

[27] H. Hdeib, “ $\omega$-closed mappings," Revista Colombiana de Matemáticas, vol. XVI 16, pp. 65-78, 1982.

[28] S. Al Ghour and S. Al-Zoubi, "A new class between theta open sets and theta omega open sets," Heliyon, vol. 7, no. 1, Article ID e05996, 2021.

[29] S. Al Ghour and B. Irshidat, "On $\theta$ continuity," Heliyon, vol. 6, no. 2, Article ID e03349, 2020.

[30] L. L. L. Butanas and M. A. Labendia, " $\theta_{\omega}$-connected space and $\theta_{\omega}$-continuity in the product space," Poincare Journal of Analysis and Applications, vol. 7, no. 1, pp. 79-88, 2020.

[31] R. M. Latif, "Theta- $\omega$ - mappings in topological spaces," WSEAS Transactions on Mathematics, vol. 19, pp. 186-207, 2020.

[32] A. Al-Omari and H. Al-Saadi, "On $\omega^{*}$-connected spaces," Songklanakarin Journal of Science and Technology, vol. 42, pp. 280-283, 2020.

[33] N. Noble, "Some thoughts on countable Lindelöf products," Topology and its Applications, vol. 259, pp. 287-310, 2019.

[34] H. H. Al-Jarrah, A. Al-Rawshdeh, E. M. Al-Saleh, and K. Y. Al-Zoubi, "Characterization of \$Rlomega $O(X)$ sets by using \$\{\elta\{lomega\}\}-\$cluster points," Novi Sad Journal of Mathematics, vol. 49, no. 2, pp. 109-122, 2019.

[35] S. Al Ghour and S. El-Issa, " $\theta \omega-$ Connectedness and $\omega-R 1$ properties,” Proyecciones, vol. 38, no. 5, pp. 921-942, 2019.

[36] C. Carpintero, N. Rajesh, and E. Rosas, "On real valued $\omega$-continuous functions," Acta Universitatis Sapientiae, Mathematica, vol. 10, no. 2, pp. 242-248, 2018.

[37] G. Al and B. Irshedat, "The topology of $\theta \omega$-open sets," Filomat, vol. 31, no. 16, pp. 5369-5377, 2017.

[38] Y. Hirata and Y. Yajima, "A characterization of the countable paracompactness for products of ordinals," Topology and its Applications, vol. 282, Article ID 107325, 2020.

[39] J. Niknejad, "Normal images of the product and countably paracompact condensation," Topology and its Applications, vol. 274, Article ID 107127, 2020.

[40] P. Szewczak and B. Tsaban, "Products of general Menger spaces," Topology and its Applications, vol. 255, pp. 41-55, 2019.

[41] S. Al Ghour, "Theorems on strong paracompactness of product spaces," Mathematical Notes, vol. 103, no. 1-2, pp. 54-58, 2018.

[42] P. Szewczak, "Productivity of paracompactness and closed images of real GO-spaces," Topology and its Applications, vol. 222, pp. 254-273, 2017.

[43] H. Junnila, "A note on productively paracompact spaces," Topology and its Applications, vol. 230, pp. 550-561, 2017. 
[44] G. Markoski and A. Buklla, "On product of spaces of quasicomponents," Filomat, vol. 31, no. 20, pp. 6307-6311, 2017.

[45] K. Alster, "On paracompactness in cartesian products and Telgarsky's game," Houston Journal of Mathematics, vol. 39, no. 4, pp. 1401-1422, 2013.

[46] R. Arens and J. Dugundji, "Remark on the concept of compactness," Portugaliae Mathematica, vol. 9, pp. 141-143, 1950.

[47] C. M. Pareek, "Hereditarily Lindelöf and hereditarily almost Lindelöf spaces," Japanese Journal of Mathematics, vol. 30, no. 4, pp. 635-639, 1985.

[48] K. Al-Zoubi and K. Al-Nashef, "The topology of $\omega$-open subsets," Al-Manarah Journal, vol. 9, pp. 169-179, 2003.

[49] R. Telgársky, "C-scattered and paracompact spaces," Fundamenta Mathematicae, vol. 73, no. 1, pp. 59-74, 1971.

[50] H. Z. Hdeib, "On the product of paracompact spaces," Questions and answers in general topology, vol. 5, pp. 229-236, 1987.

[51] R. L. Ellis, "Extending continuous functions on zero-dimensional spaces," Mathematische Annalen, vol. 186, no. 2, pp. 114-122, 1970.

[52] D. K. Burke, "Covering properties," in Handbook of SetTheoretic Topology, K. Kunen and J. E. vaughan, Eds., pp. 347-422, Amsterdam, Netherlands, 1984. 